\title{
Dosis alternativas del tinidazol en el tratamiento de la vaginosis bacteriana: Reporte preliminar.
}

\author{
José Núñez Troconis \\ Departamento de Obstetricia y Ginecología. Facultad de Medicina. Universidad \\ del Zulia. Maracaibo, Venezuela.
}

Palabras elave: tinidazol; vaginosis bacteriana; Gardnerella vaginalis.

Resumen: Los derivados imidazólicos como el metronidazol y el secnidazol han demostrado funcionar en la curación de la vaginosis bacteriana (VB), pero sus efectos secundarios y modo de administración son de gran interés. El objetivo del presente estudio fue determinar si el tinidazol en diferentes dosis también es un medicamento eficaz para la curación de la VB. Se distribuyeron al azar 45 pacientes con diaǵnóstico de VB en tres grupos, a los cuales se les administró tinidazol por vía oral (VO) en diferentes dosis: grupo A $(\mathrm{n}=9): 1 \mathrm{~g}$ por un día; grupo $\mathrm{B}(\mathrm{n}=17)$ : $2 \mathrm{~g}$ por un día; grupo $\mathrm{C}(\mathrm{n}=19)$ : $2 \mathrm{~g}$ por dos días. El grupo B mostró curación clínica del 82,4\% ( $<<0,0001$ y la citológica fue de $94,1 \%(\mathrm{p}=0,0001)$. En el grupo $\mathrm{C}$ se encontró que la curación clínica fue del $84,2 \%(\mathrm{p}<0,0001)$ y la citológica fue de $89,5 \%(\mathrm{p}<0,0001)$. El uso único de $1 \mathrm{~g}$ no fue suficiente para lograr la curación clínica $(55,6 \%)$ y/o citológica $(66,7 \%)$. En relación a los efectos secundarios, no se encontró ninguna ventaja con el uso de dosis más bajas y por menor tiempo. La conclusión de los resultados preliminares del presente trabajo fue que el uso de $2 \mathrm{~g}$ VO de tinidazol por un día fue tan eficaz para la curación de la VB como el uso de $2 \mathrm{~g}$ por dos días.

Autor de correspondencia: José Núñez Troconis. Departamento de Obstetricia y Ginecología. Facultad de Medicina. Universidad del Zulia. Maracaibo, Venezuela. Correo electrónico: jtnunezt@gmail.com 


\title{
Alternative doses of tinidazole in the treatment of bacterial vaginosis: Preliminary report.
}

\author{
Invest Clin 2020; 61 (1): 7-20
}

Key words: tinidazole; bacterial vaginosis; Gardnerella vaginalis.

\begin{abstract}
Imidazole derivatives such metronidazole and secnidazole have shown to work in treating bacterial vaginosis (BV) but their side effects and mode of administration are of concern. The aim of the present study was to determine whether tinidazole in different doses was also an effective therapy for BV. Forty-five patients with diagnosis of BV were randomized in three groups who received tinidazol orally in different dosages: group $\mathrm{A}(\mathrm{n}=9): 1 \mathrm{~g}$ for one day; group B $(n=17): 2 \mathrm{~g}$ for one day; group $\mathrm{C}(\mathrm{n}=19): 2 \mathrm{~g}$ for two days. The group B showed an $82.4 \%$ clinical cure $(\mathrm{p}<0.0001)$ and the cytological was $94.1 \%(p=0.0001)$. We found a clinical cure of $84.2 \%(p<0.0001)$ and the cytological cure was $85.5 \%(\mathrm{p}<0.0001)$ in the group C. One gram (Group A) was not enough to achieve the clinical and cytological cure. There were no dif-ferences in side effects using lower doses and less time of treatment. The pre-liminary conclusion was that $2 \mathrm{~g}$ of tinidazol for one day had the same efficacy to cure BV that $2 \mathrm{~g}$ for two days.
\end{abstract}

Recibido: 06-09-2018 Aceptado: 19-09-2019

\section{INTRODUCCIÓN}

El flujo vaginal es una de las causas más frecuentes de consulta ginecológica en mujeres en edad reproductiva y es responsable de 5 a 10 millones de visitas ginecológicas por año a nivel mundial (1). La vaginosis bacteriana (VB) representa la causa más común de flujo vaginal que afecta del 5 al $58 \%$ de las mujeres en edad reproductiva a nivel mundial (2-4). La VB se caracteriza por un flujo homogéneo, blanco-grisáceo y de mal olor producido por el incremento de la producción de aminas como putrecina, cadaverina y trimetilamina. La producción de estas aminas es ocasionada por un sobrecrecimiento de las bacterias anaeróbicas, en especial Gardnerella vaginalis (Gv), las cuales contribuyen a elevar el $\mathrm{pH}$ vaginal $(>4,5)$ y determinan la presencia de células claves $(5,6)$, con o sin síntomas y signos de inflamación vulvo-vagiinal $(7,8)$.
La VB es un factor de riesgo y de morbilidad para la aparición de enfermedad inflamatoria pélvica, infertilidad, síndrome uretral, vulvo-vaginitis, cervicitis, celulitis de cúpula vaǵinal, aborto, corioamionitis, ruptura prematura de membranas con el desencadenamiento de trabajo de parto prematuro, niños con bajo peso al nacer, y aumento de la susceptibilidad a la adquisición de enfermedades de transmisión sexual producidas por el virus del herpes simple 2, el virus del papiloma humano, gonococo, Chlamydia tracomatis, Tricomona vaginalis $\mathrm{y}$, en especial, el virus de la inmunodeficiencia adquirida (9-12).

La VB puede ser diagnosticada clínicamente usando los criterios de Amsel (13) o con la coloración de Gram, considerada como la prueba de laboratorio perfecta para diaǵnosticarla (Criterios de Nugent). Con esta última, se puede determinar la concentración de lactobacilos, bacterias Gram negativas y positivas, bacterias tipo 
cocos como Gv, Prevotella, Porphyromonas, peptoestreptococo, peptococos, etc., y bacterias curvas Gram negativas como Mobiluncus (12). Actualmente, están disponibles otras pruebas como el Affirm VP III (Becton Dickinson, Spark, USA); esta prueba usa una sonda de hibridización del ADN, la cual detecta concentraciones elevadas de GV y la prueba OSOM Blue (Sekisui Diagnostics, Framingham, USA) la cual detecta sialidiasa en la secreción vaginal y cuyos resultados son comparables con la tinción de Gram. Otra prueba disponible actualmente es la de la prolineaminopeptidasa que permite la medición del $\mathrm{pH}$ vaginal y de la amina trimetilamina; sin embargo, su sensibilidad y especificidad es baja por lo que el Centro para el Control de Enfermedades y Prevención (CDC, por sus siǵlas en inglés) no la recomienda. La reacción en cadena de la polimerasa ha sido usada para determinar la presencia de microorganismos asociados a la VB, pero su utilidad clínica está todavía por establecerse. El cultivo de la Gv no es recomendado como método de diagnnóstico debido a que no es específico. La citología cervico-vaginal (CGV) tiene una especificidad del 95\% y una sensibilidad del 50\% en el diagnóstico de la VB (14). Este es un método confiable en el diagnóstico de la $\mathrm{VB}$; sin embargo, la ausencia en la CCV no descarta la presencia de esta patología (14).

La administración del metronidazol por vía oral ha sido el tratamiento de elección de la $\mathrm{VB}$, igualmente el uso vaginal de metronidazol y clindamicina ha sido empleado ampliamente (8). El CDC recomienda el uso de diferentes medicamentos y esquemas de tratamiento en mujeres no gestantes con diagnóstico de VB (12). Como drogas y esquemas de primera línea o elección, este centro recomienda lo siguiente: metronidazol a dosis de $500 \mathrm{mg}$ vía oral (VO) 2 veces al día (BID) por 7 días o en gel a $0,75 \%$, una aplicación ( $5 \mathrm{~g}$ ) intravaginal diaria por 5 días o clindamicina en crema al $2 \%$, una aplicación (5 g) intravaginal diaria por 7 días. También, recomienda otras drogas y esquemas alter- nativos tales como: tinidazol a dosis de $2 \mathrm{~g}$ VO OD por 2 días o $1 \mathrm{~g}$ VO OD por 5 días o clindamicina a $300 \mathrm{mg}$ VO BID por 7 días o en óvulos de $100 \mathrm{mg}$ intravaginal OD por 3 días. En relación a otro derivado imidazólico que es usado en el tratamiento de la VB como es el secnidazol, se ha demostrado que el uso de $2 g(14,15)$ y de $1 g$ en una sola dosis $(15,16)$ son esquemas terapéuticos adecuados para tratar y curar la VB. En general, los derivados imidazólicos son empleados en diferentes patologías, pero debido a sus efectos secundarios, se ha buscado disminuir la dosis y el tiempo de administración (pero manteniendo su acción terapéutica), para minimizar sus efectos secundarios y permitir el cumplimiento del tratamiento.

El objetivo de la presente investigación fue determinar si el uso de dosis más bajas y/o el menor tiempo de administración del tinidazol es una alternativa para curar la VB.

\section{PACIENTES Y MÉTODO}

Se estudiaron 57 pacientes que acudieron a las consultas de Ginecología y de Patología de Cuello Uterino del Hospital "Dr. Manuel Noriega Trigo", perteneciente al Instituto Venezolano de los Seguros Sociales, Maracaibo, Venezuela. El comité ético del hospital y de la Facultad de Medicina, Universidad del Zulia, Maracaibo, Venezuela, aprobaron el estudio. A cada paciente se le explicó el objetivo del estudio y la confidencialidad del mismo, previo a la firma del consentimiento.

Los criterios de inclusión fueron: tener flujo vaginal y que reuniese 3 de 4 de los criterios de Amsel para VB (13) y/o una citología vaginal que mostrara la presencia de la Gv. Los eriterios de exclusión fueron: embarazo o lactancia, edad menor de 18 años, ingestión de antibiótico al menos 4 semanas previas la entrevista y alergía al metronidazol o sus derivados.

Después de la firma del consentimiento, se realizó una historia clínica donde se recogieron datos o antecedentes demográficos, 
gineco-obstétricos, sexuales y la clínica que presentaba. Igualmente, se le explicó a cada paciente incluida en el estudio que ellas padecían una entidad ginecológica denominada VB y que la investigación tenía por objeto estudiar el medicamento llamado tinidazol, utilizado en el tratamiento de dicha patología vaginal en una dosis menor y administrado por un período de tiempo menor, al recomendado para lograr su curación.

Posteriormente se procedió al examen ginecológico, previa colocación de un espéculo bivalvo no lubricado. Se observó la cantidad y el color del flujo vaginal, igualmente, si presentaba olor fétido espontáneo. Se procedió a medir el $\mathrm{pH}$ vaginal usando una tira de papel especial para medirlo (Papers Hydron, Micro Essential Laboratory, Brooklyn, NY, USA), colocándolo a nivel de una de las paredes laterales o fondo de saco anterior de la vagina. Así mismo, se tomaron dos muestras de la secreción, las cuales se extendieron en láminas porta-objeto: a la primera se le colocó una gota de azul de metileno para su estudio al fresco y a la segunda se le colocó una gota de $\mathrm{KOH}$ para la prueba de Whiff o de las aminas. Posteriormente, se procedió a la toma de muestras del fondo de saco posterior, exocervix y endocervix para la citología cervico-vaginal o Papanicolaou. Cada paciente fue interrogada y examinada por el autor durante su primera y segunda visita.

Las pacientes se distribuyeron al azar en 3 grupos de acuerdo al número terminal de su documento de identificación: Grupo A (1$3)$; grupo B (4-6) y grupo C (7-0). Las pacientes del grupo A recibieron 1 g VO por 1 día; las del grupo B $1 \mathrm{~g}$ VO OD por 2 días y las del grupo $\mathrm{C} 2 \mathrm{~g}$ VO OD por 2 días. Este último es el esquema de tratamiento recomendado por el CDC para el tratamiento para la VB (12).

A cada paciente se le instruyó no realizarse duchas vaginales, no tomar otro tipo de antibiótico y no tener relaciones sexuales; si llegase a tenerlas se le explicó que debían ser protegidas con preservativos o darle el tratamiento de tinidazol a su pareja antes de la relación sexual.
Las pacientes fueron citadas para la semana siguiente para realizar su control y examen clínico similar a la primera visita y determinar si se consiguió la curación clínica y/o citológica. Se consideró curación clínica basada en los criterios de Amsel (13) (flujo, prueba de $\mathrm{KOH}$, pH y estudio al fresco), al no encontrarse 3 de estos 4 criterios (17) y curación citológica al observarse ausencia de la Gv.

Los datos obtenidos fueron descarǵados y guardados usando el programa Excel 2010 (Microsoft Co Redmond, WA) y luego trasladados al programa SPSS para Windows versión 20 (IBM, Armonk, NY, USA). Se calculó el promedio y la desviación estándar de las variables continuas. Las variables categóricas fueron expresadas en porcentaje, así como también la frecuencia simple. La prueba de $\mathrm{T}$ para muestras pareadas y para una sola muestra, la prueba de Chi Cuadrado de Pearson y la prueba Exacta de Fisher fueron empleadas para comparar las variables durante la primera y segunda visita; así mismo, se emplearon las tablas cruzadas 2x2 con el cálculo de Odd Ratio (OD) y el Intervalo de Confianza del 95\% (IC 95\%) para comparar los grupos estudiados. Se consideró el valor de $\mathrm{p}<\mathrm{de}$ 0,05 como estadísticamente significativo.

\section{RESULTADOS}

Se estudiaron y completaron 45 de 57 pacientes con diagnóstico de $\mathrm{VB}$, las cuales fueron distribuidas en 3 diferentes grupos. Doce mujeres fueron excluidas por no haber regresado a la visita del control post-tratamiento: 2 pertenecieron al grupo A, 5 al grupo B y 5 al grupo C. La segunda visita o control post-tratamiento, se realizó en 11,96 $\pm 9,17$ días (mediana:8; rango:7-47).

La edad promedio fue $39,62 \pm 9,6$ años (media \pm DS; mediana:40 años;rango: 8-61). Treinta y ocho $(84,4 \%)$ de las 45 estudiadas mencionaron haber estado embarazada; el promedio de embarazos fue de 3,05 \pm 2 DS (mediana: 2;rango: $1-8)$. Veintiocho $(73,7 \%)$ 
de las 38 que habían estado embarazadas, reportaron haber tenido partos y el promedio de partos fue de 2,86 $\pm 1,35$ DS (mediana: 3;rango: 1 -5 ). En la Tabla I se observan dichas variables en cada grupo estudiado. Trece pacientes $(34,2 \%)$ mencionaron que se le había practicado cesárea.

La edad promedio de la primera relación sexual fue de 18,53 $\pm 3,59$ DS años (mediana: 18 años; ranǵo: 13-32). El número promedio de compañeros sexuales fue de 2,22 $\pm 1,12$ DS (mediana: 2; rango:1-5). La Tabla I muestra dichas variables para cada grupo. Veinte $(44,4 \%)$ mujeres usaban alguno método de anticoncepción, siendo las píldoras anticonceptivas orales, el método más usado $(\mathrm{n}=11 ; 24,4 \%)$.

La distribución por grupo tras la exclusión de aquellas doce mujeres que no se presentaron a la visita post-tratamiento fue la siguiente: Grupo A: 9 (20\%), grupo B: 17 $(37,8 \%)$ y grupo C: $19(42,2 \%)$.

Cuarenta y dos $(83,3 \%)$ se incluyeron por presentar clínica de $\mathrm{VB}$, que reunieron al menos 3 de los 4 criterios de Amsel y las 3 restantes fueron incluidas por la presencia de la Gr en la GCV. La presencia de flujo vaginal fue mencionada por 8 pacientes (88,9\%) del grupo A, 17 (100\%) del grupo B y 17 (89,5\%) del grupo C. El promedio de aparición o presencia del flujo vaginal fue de 29,17 \pm 52 semanas (mediana:8,5 semanas; rango: 2 días-257 semanas). El cincuenta y ocho por ciento $(n=26)$ de ellas reportaron que el flujo era de moderada a abundante cantidad. De las 42 mujeres con manifestaciones clínicas, $14 \quad(33,3 \%)$ reportaron prurito vulvar, 5 (11,9\%) prurito vaginal, 5 $(11,9 \%)$ dispaurenia, $22(52,4 \%)$ disuria y 16 $(38,1 \%)$ fetidez espontánea y después de las relaciones sexuales. El promedio de aparición del prurito vulvar fue de $11,4 \pm 13,3$ semanas (mediana: 8,5 semanas; rango: 2 días - 51,4 semanas). El promedio de aparición del purito vaginal fue de $10,4 \pm 9,4$ semanas (mediana: 6,4 semanas;rango: 20 días -25,7 semanas). Treinta y seis $(80 \%)$ de las 45 mencionaron que eran sexualmente activas $\mathrm{y}$ todas reportaron tener el mismo compañero sexual.

En el primer examen clínico, se encontró flujo vaǵinal en las 45 pacientes estudiadas, 31 de ellas $(68,9 \%)$ lo presentaron en escasa cantidad. La Tabla I muestra la comparación de los hallazgoos relativos al flujo vaginal entre los 3 grupos durante la primera visita.

En la segunda visita de las 42 pacientes que reportaron flujo durante la primera visita, $24(57,1 \% ; p=0,0001)$ mencionaron que el flujo vaginal desapareció después del tratamiento, de las cuales 5 de las 8 (62,5\%; $\mathrm{p}=0,013)$ fueron del grupo A, 9 de 17 (53\%; p<0,001) del grupo B y 10 de $17 \quad(59 \%$; $\mathrm{p}<0,001)$ del grupo C. Doce (67\%) de las 18 que reportaron la persistencia del flujo, mencionaron que fue de escasa cantidad. El prurito vulvar desapareció en 9 de $14 \mathrm{mu}$ jeres $(64,3 \% ; p=0,002)$ que lo reportaron durante la primera visita mientras que el prurito vaginal se mantuvo presente en 1 de las $5(20 \%, \mathrm{p}=\mathrm{NS})$ que lo reportaron inicialmente, pero 2 casos presentaron el prurito vaginal después del tratamiento. La dispaurenia y la disuria persistieron en 2 pacientes $(4,4 \% ; \mathrm{p}=\mathrm{NS})$.

En el segundo examen o de control post-tratamiento, se encontró flujo vaǵinal en 35 de las 45 pacientes $(77,8 \%, p=0,001)$ y la distribución de casos por grupos fue la siguiente: 7 de $9(77,8 \%, p=N S)$ en el grupo A, 13 de $17(76,5 \%, p=0,041)$ en el grupo B y 15 de $19(78,9 \%, \mathrm{p}=0,042)$ en el grupo $\mathrm{C}$. En 24 mujeres $(53,3 \%)$, el flujo fue escaso. En $20(57,1 \%)$, el flujo fue de color blancogrisáceo, siendo estadísticamente significativa la mejoría $(\mathrm{p}=0,032)$, al compararse con el número de pacientes $(n=33)$ que presentaron este color en el primer examen; sin embargo, al compararse esta variable en cada grupo, se observaron los siguientes hallazgos: grupo A: 3 de 7 (42,9\%, p=NS), grupo B: 8 de $13(61,5 \%, p=N S)$ y grupo $\mathrm{C}$ : 9 de $15(60 \%, \mathrm{p}=\mathrm{NS})$. En 27 de las 35 mujeres que inicialmente se presentaron con flujo vaginal $(77,1 \%)$, se mantuvo el aspec-

Vol. 61(1): 5 - 18, 2020 
TABLA I

DATOS DEMOGRÁFICOS Y HALLAZGOS CLÍNICOS Y DE LABORATORIO EN LA PRIMERA VISITA

\begin{tabular}{|c|c|c|c|c|c|c|}
\hline \multirow{2}{*}{ Variable } & \multicolumn{2}{|c|}{ Grupo A $(n=9)$} & \multicolumn{2}{|c|}{ Grupo B $\quad(n=17)$} & \multicolumn{2}{|c|}{ Grupo C $(n=19)$} \\
\hline & $\mathrm{X} \pm \mathrm{DE}$ & $\%(\mathrm{n})$ & $\mathrm{X} \pm \mathrm{DE}$ & $\%(\mathrm{n})$ & $\mathrm{X} \pm \mathrm{DE}$ & $\%(\mathrm{n})$ \\
\hline \multirow{2}{*}{ Edad (años) } & $37,33 \pm 11,7$ & & $40,82 \pm 9,52$ & & $39,63 \pm 9$ & \\
\hline & Rango:18-51 & & Rango: 20-61 & & Rango: 20-49 & \\
\hline \multicolumn{7}{|l|}{ Edad de primera } \\
\hline relación sexual (años) & $18,67 \pm 3,8$ & & $17,58 \pm 2,87$ & & $19,31 \pm 4$ & \\
\hline Número de embarazos & $3,43 \pm 2,57$ & & $2,65 \pm 1,36$ & & $2,62 \pm 1,85$ & \\
\hline Número de partos & $3,4 \pm 1,67$ & & $1,94 \pm 1,63$ & & $2,72 \pm 1,42$ & \\
\hline Compañeros sexuales & $1,88 \pm 0,78$ & & $2,35 \pm 1,36$ & & $2,26 \pm 1$ & \\
\hline Manifestaciones clínicas & & $88,9(8)$ & & $100(17)$ & & $89,5(17)$ \\
\hline Flujo & & $100(9)$ & & $100(17)$ & & $100(19)$ \\
\hline Cantidad & & $44,4(4)$ & & $29,4(5)$ & & $26,3(5)$ \\
\hline Consistencia homogénea & & $100(9)$ & & $100(17)$ & & $88,9(15)$ \\
\hline Color (Blanco-grisáceo) & & $55,6(5)$ & & $47,1(8)$ & & $47,4(9)$ \\
\hline Fetidez & & $100(9)$ & & $70,6(12)$ & & $68,4(13)$ \\
\hline $\mathrm{KOH}$ & & $100(9)$ & & $94,1(16)$ & & $89,5(17)$ \\
\hline pH 5> & & $100(9)$ & & $100(17)$ & & $100(19)$ \\
\hline Freseo (Gv/Células Clue) & & $88,9(6)$ & & $88,2(15)$ & & $88,5(17)$ \\
\hline Citología vaginal (Gv) & & $77,8(7)$ & & $76,5(13)$ & & $78,9(15)$ \\
\hline
\end{tabular}

Los resultados se expresan en $\mathrm{X} \pm$ desviación estándar y poreentajes.

Los números entre paréntesis representa el número de casos.

Gv: Gardnerella vaginalis

Cantidad: moderada/abundante.

to homogéneo y no se encontró diferencia estadística significativa en esta variable, al comparar los resultados de la primera y segunda visita y los resultados de cada grupo. Solo 6 mujeres $(17,1 \%)$ presentaron fetidez del flujo $(p=0,0001), 2$ pacientes en cada grupo estudiado. La prueba de $\mathrm{KOH}$ fue negativa en $36(80 \%)$ del total de pacientes $(p=0,0001)$ y al estudiarse esta variable por grupo se observó este mismo resultado en el grupo A, 4 de $9(44,4 \%, p=0,035)$, grupo $B$, 14 de $17(82,4 \%, \mathrm{p}=0,0001)$ y grupo $\mathrm{C}, 18$ de $19(94,7 \%, \mathrm{p}=0,0001)$. El $\mathrm{pH}$ fue 4 en 22 de las 45 pacientes estudiadas (49\%), siendo significativo estadísticamente el descenso del $\mathrm{pH}(\mathrm{p}=0,0001)$, mientras que por grupos se observó 1 de $9(11,1 \%, p=N S)$ en el grupo A, 12 de $17(70,6 \%, p=0,0001)$ en el grupo B y 9 de $19(47,4 \%, p=0,0001)$ en el grupo C. Cuando se analizó el estudio al fresco de la visita de control, la Gv y/o células clue (CC) se encontraron en solo 10 de las
45 pacientes $(22,2 \%)$, siendo este hallazgo estadísticamente significativo $(\mathrm{p}=0,0001)$. En el grupo A, 8 de las 9 mujeres $(88,9 \%)$ en quienes se encontró $\mathrm{VB}$ en la primera visita, se detectó Gv/CG en solo $6(66,7 \%, \mathrm{p}=\mathrm{NS})$ durante la visita de control. De las 17 pacientes correspondientes al grupo B, 15 (88,2\%) que presentaron Gv/CC al fresco, en ninguna de ellas se pudo demostrar su presencia posterior al tratamiento, siendo estadísticamente significativa la respuesta terapéutica $(\mathrm{p}=0,0001)$. En el grupo C, $17(88,5 \%)$ de 19 que resultaron positivas para la presencia de Gv y/o CC durante la primera visita, posteriormente al tratamiento, $17(88,5 \%)$ de ellas resultaron negativas $(p=0,0001)$. Así mismo, de las 40 pacientes con la presencia de signos de VB en el freseo durante la primera visita, en 10 de ellas (25\%) persistió la VB en la segunda visita $(p=0,0001)$. La CV de control mostró en 7 de las 45 mujeres $(15,6 \%)$ la presencia de la Gv $(p=0,0001)$ : 
grupo A: 4 CV (44,4\%, p=NS), grupo B: 1 CV $(5,9 \%, p=0,0001)$ y grupo C: 2 CV $(28,6 \%$, $\mathrm{p}=0,0001)$. De las 10 pacientes donde el fresco mostro signos de $\mathrm{VB}$, la CV reportó la presencia de la Gv en 6 de ellas $(85,7 \%)$. En 35 pacientes cuya CV mostró la presencia de la Gv durante la primera visita, en 7 de ellas persistió la presencia de la bacteria. Solo un caso de los 7 (14,3\%) que la CV detectó.

En el Grupo A no se consiguió curación clínica en 5 de las 9 pacientes $(55,6 \%$, $\mathrm{p}=\mathrm{NS}$; OR 0,444 IC 95\%: 0,4-0,85). Sin embargo, aunque en 5 de las 9 mujeres se obtuvo curación citológica $(55,6 \%, \mathrm{p}=\mathrm{NS}$; OR: 0,333 IC 95\%:0,51-0,718), al comparar ambas curaciones, no se encontró significancia estadística $(p=0,167$, OR: 0,400 IC 95\%: 0,137-1,170).

$\mathrm{Al}$ analizar el grupo B, se halló que hubo curación clínica en 14 de las 17 pacientes $(82,4 \%, p=0,0001$; OR: 0,824 IC 95\%: 0,62$1,03)$ y curación citológica en 16 de las 17 pacientes $(94,1 \% ; p=0,0001 ;$ OR: $0,941, \mathrm{IC}$ 95\%: 0,82-1,07). Al comparar ambas curaciones no se encontró ninguna diferencia significativa ( $\mathrm{p}=0,172$; OR: 0,667 , IC 95\%: 0,33-1,48).

El Grupo G mostró curación clínica en 16 de las 19 pacientes (84,2\%: p=0,0001; OR: 0,842 IC 95\%: 0,66-1,02) y una curación citológica del 89,5\% $(\mathrm{n}=17 ; \mathrm{p}=0,0001$; OR: 0,895 IC 95\%: 0,74-1,05). No se encontró ninguna diferencia estadísticamente significativa al comparar las dos curaciones $(p=0,331$; OR: 0,333 IC 95\%: 0,067-1,657).

Si se comparan los resultados obtenidos durante el segundo examen entre los grupos para determinar la efectividad curativa de las dosis y el tiempo de administración (ver Tablas II, III, IV), se observa que se halló curación clínica y citológica en los grupos $\mathrm{B}$ y $\mathrm{C}$, no así en el grupo A. Al comparar los grupos $\mathrm{B}$ y $\mathrm{C}$, no se encontró ninguna diferencia estadística.

El cincuenta y seis por ciento $(n=25)$ de las pacientes reportaron efectos secundarios; siendo el sabor metálico el más frecuente, reportado en el $88 \%(n=22)$ de las
25 mujeres. Otros efectos secundarios fueron náuseas, mareos, vómitos, cefalea y pirosis. Cinco de las 9 mujeres $(55,6 \%)$ del grupo A, 9 de 17 (52,9\%) del grupo B y 11 de 19 (57,9\%) del grupo $\mathrm{C}$ reportaron efectos secundarios. En relación a los efectos secundarios, no se encontró ninguna diferencia estadística al comparar los 3 grupos entre sí (Grupo A vs grupo B: p=0,899, OR: 1,049 IC 95\%: 0,50-2,19; grupo A vs grupo C: $p=0,907$, OR: 0,960 IC 95\%: 0,47-1,93); grupo B vs grupo $\mathrm{C}: \mathrm{p}=0,765$, OR: 0,914 IC 95\%: 0,50-1,64).

Cinco de las 45 pacientes $(11,1 \%)$ tuvieron relaciones sexuales durante el estudio. Todas usaron algún tipo de protección: 4 usaron preservativos y una recibió tratamiento junto a su pareja.

\section{DISCUSIÓN}

La VB ha sido tratada de diferentes formas y medicamentos con relativos buenos resultados $(7,15,18-21)$. Entre esos medicamentos están los derivados 5-nitroimidazolicos tales como el metronidazol, ornidazol, nimorazol, secnidazol y tinidazol (16). Uno de esos derivados 5-nitroimidazolicos, el tinidazol (1-[2-(etilsulfonil) etil]-2-metil-5nitroimidazol) está estructuralmente relacionado con el metronidazol y el secnidazol (8).

El tinidazol fue usado en el tratamiento de la tricomoniasis vaginal por primera vez en 1967, siendo empleado en Europa desde hace varias décadas. Así mismo, ha sido usado en el tratamiento de otras infecciones producidas por protozoarios incluyendo la amibiasis y la giardiasis, en el tratamiento de las infeceiones bacterianas como el Helicobacter pylori, así mismo en infecciones producidas por bacterias anaeróbicas tales como infecciones intraoperatorias y post-operatorias, infecciones de heridas operatorias de piel y tejidos blandos y por supuesto infecciones ginecológicas tales como la VB (8). La vida media del tinidazol es de 12,7 horas, por lo tanto, con una vida media más prolongada que 
el metronidazol y con una concentración plasmática antimicrobiana que la mantiene en los tejidos lo suficiente para permi- tir que su administración sea una vez al día (8), además que produce menos efectos secundarios que el metronidazol (17).

\section{TABLA II}

COMPARACIÓN ENTRE EL GRUPO AYB EN EL $2^{\circ}$ EXAMEN

\begin{tabular}{|c|c|c|c|c|}
\hline & Grupo A & Grupo B & & \\
\hline & $\mathrm{n}(\%)$ & $\mathrm{n}(\%)$ & Siǵnificancia & OR (IC 95\%) \\
\hline \multicolumn{5}{|l|}{ Flujo } \\
\hline Sí & $7(77,8)$ & $13(76,5)$ & 0,940 & $0,944(0,212-4.2 .2$ \\
\hline No & $2(22,2)$ & $4(23,5)$ & & \\
\hline Total & $9(100)$ & $17(100)$ & & \\
\hline \multicolumn{5}{|l|}{ Color } \\
\hline Blanco-grisáceo & $3(42,9)$ & $8(61,5)$ & 0,423 & $0,696(0,267-1,814)$ \\
\hline Otro & $4(57,1)$ & $5(38,5)$ & & \\
\hline Total & $7(100)$ & $13(100)$ & & \\
\hline \multicolumn{5}{|l|}{ Consistencia } \\
\hline Homogénea & $5(71,4)$ & $10(76,9)$ & 0,787 & $0,929(0,533-1,623)$ \\
\hline Otra & $2(28,6)$ & $3(23,1)$ & & \\
\hline Total & $7(100)$ & $13(100)$ & & \\
\hline \multicolumn{5}{|l|}{ Fetidez (mal color) } \\
\hline Sí & $2(28,6)$ & $2(15,4)$ & 0,482 & $0,844(0,501-1,424)$ \\
\hline No & $5(71,4)$ & $11(84,6)$ & & \\
\hline Total & $7(100)$ & $13(100)$ & & \\
\hline \multicolumn{5}{|l|}{$\mathrm{KOH}$} \\
\hline Positivo & $5(55,6)$ & $3(17,6)$ & $<0,04$ & $3,141(0,966-10,261)$ \\
\hline Negativo & $4(44,4)$ & $14(82,4)$ & & \\
\hline Total & $9(100)$ & $17(100)$ & & \\
\hline \multicolumn{5}{|l|}{$\mathrm{pH}$} \\
\hline$<4,5$ & $1(11,1)$ & $12(70,6)$ & $<0,004$ & $3,022(1,347-6,539)$ \\
\hline$>4,5$ & $8(88,9)$ & $5(29,4)$ & & \\
\hline Total & $9(100)$ & $17(100)$ & & \\
\hline \multicolumn{5}{|l|}{ Estudio al Fresco } \\
\hline Células Clue/Gv & $6(66,7)$ & $2(11,8)$ & $<0,004$ & $5,667(1,424-22,556)$ \\
\hline Otros hallazgos & $3(33,3)$ & $15(88,2)$ & & \\
\hline Total & $9(100)$ & $17(100)$ & & \\
\hline \multicolumn{5}{|l|}{ Cítologia Vaǵinal } \\
\hline Gv & $4(44,4)$ & $1(5,9)$ & $<0,01$ & $7,556(0,985-57,925)$ \\
\hline Otros hallazgos & $5(55,6)$ & $16(94,1)$ & & \\
\hline Total & $9(100)$ & $17(100)$ & & \\
\hline
\end{tabular}

Grupo A: 1 g por un día; grupo B: 2 g por 1 día. 
TABLA III

COMPARACIÓN ENTRE EL GRUPO A Y C EN EL $2^{\circ}$ EXAMEN

\begin{tabular}{|c|c|c|c|c|}
\hline & Grupo A & Grupo C & & \\
\hline & n (\%) & n (\%) & Siǵnificancia & OR (IC 95\%) \\
\hline \multicolumn{5}{|l|}{ Flujo } \\
\hline Sí & $7(77,8)$ & $15(78,9)$ & 0,944 & $0,985(0,648-1,498)$ \\
\hline No & $2(22,2)$ & $4(21,1)$ & & \\
\hline Total & $9(100)$ & $19(100)$ & & \\
\hline \multicolumn{5}{|l|}{ Color } \\
\hline Blanco-grisáceo & $3(42,9)$ & $9(60)$ & 0,452 & $0,714(0,276-1,847)$ \\
\hline Otro & $4(57,1)$ & $6(40)$ & & \\
\hline Total & $7(100)$ & $15(100)$ & & \\
\hline \multicolumn{5}{|l|}{ Consistencia } \\
\hline Homogénea & $5(71,4)$ & $12(80)$ & 0,655 & $0,893(0,524-1,521)$ \\
\hline Otra & $2(28,6)$ & $3(20)$ & & \\
\hline Total & $7(100)$ & $15(100)$ & & \\
\hline \multicolumn{5}{|l|}{ Fetidez(mal olor) } \\
\hline Sí & $2(28,6)$ & $2(13,3)$ & 0,398 & $0,824(0,495-1,371)$ \\
\hline No & $5(71,4)$ & $13(86,7)$ & & \\
\hline Total & $7(100)$ & $15(100)$ & & \\
\hline \multicolumn{5}{|l|}{$\mathrm{KOH}$} \\
\hline Positivo & $5(55,6)$ & $1(5,3)$ & $<0,002$ & $10,556(1,435-77,620)$ \\
\hline Negativo & $4(44,4)$ & $18(94,7)$ & & \\
\hline Total & $9(100)$ & 19(100) & & \\
\hline \multicolumn{5}{|l|}{$\mathrm{pH}$} \\
\hline$<4,5$ & $1(11,1)$ & $9(47,2)$ & $<0,05$ & $1,689(1,040-2,743)$ \\
\hline$>4,5$ & $8(88,9)$ & $10(52,6)$ & & \\
\hline Total & $9(100)$ & 19(100) & & \\
\hline \multicolumn{5}{|l|}{ Estudio al Freseo } \\
\hline Células Clue/Gv & $6(66,7)$ & $2(10,5)$ & $<0,002$ & $6,333(1,578-25,426)$ \\
\hline Otros hallazgoos & $3(33,3)$ & $17(89,5)$ & & \\
\hline Total & $9(100)$ & 19(100) & & \\
\hline \multicolumn{5}{|l|}{ Citología Vaginal } \\
\hline GV & $4(44,4)$ & $2(10,5)$ & $<0,04$ & $4,222(0,941-18,936)$ \\
\hline Otros hallazgos & $5(55,6)$ & $17(89,5)$ & & \\
\hline Total & $9(100)$ & $19(100)$ & & \\
\hline
\end{tabular}

Grupo A: 1 g por un día; Grupo C: 2 g por dos días. 
TABLA IV

COMPARACIÓN ENTRE EL GRUPO B Y C EN EL 2 EXAMEN

\begin{tabular}{|c|c|c|c|c|}
\hline & Grupo B & Grupo C & & \\
\hline & n (\%) & n (\%) & Siǵnificancia & OR (IC 95\%) \\
\hline \multicolumn{5}{|l|}{ Flujo } \\
\hline Sí & $13(76,5)$ & $15(78,9)$ & 0,858 & $0,969(0,682-1,376)$ \\
\hline No & $4(23,5)$ & $4(21,1)$ & & \\
\hline Total & $17(100)$ & $19(100)$ & & \\
\hline \multicolumn{5}{|l|}{ Color } \\
\hline Blanco-ǵrisáceo & $8(61,5)$ & $9(60)$ & 0,199 & $0,479(0,147-1,565)$ \\
\hline Otro & $5((38,5)$ & $6(40)$ & & \\
\hline Total & $13(100)$ & $15(100)$ & & \\
\hline \multicolumn{5}{|l|}{ Consistencia } \\
\hline Homogénea & $10(76,9)$ & $12(80)$ & 0,843 & $0,962(0,651-1,421)$ \\
\hline Otra & $3(23,1)$ & $3(20)$ & & \\
\hline Total & $13(100)$ & $15(100)$ & & \\
\hline \multicolumn{5}{|l|}{ Fetidez(mal olor) } \\
\hline Sí & $2(15,4)$ & $2(13,3)$ & 0,877 & $0,976(0,720-1,325)$ \\
\hline No & $11(84,6)$ & $13(86,7)$ & & \\
\hline Total & 13(100) & $15(100)$ & & \\
\hline \multicolumn{5}{|l|}{$\mathrm{KOH}$} \\
\hline Positivo & $3(17,6)$ & $1(5,3)$ & 0,615 & $0,559(0,055-5,627)$ \\
\hline Negativo & $14(82,4)$ & $18(94,7)$ & & \\
\hline Total & $17(100)$ & $19(100)$ & & \\
\hline \multicolumn{5}{|l|}{$\mathrm{pH}$} \\
\hline$<4,5$ & $12(70,6)$ & $9(47,2)$ & 0,158 & $0,559(0,239-1,309)$ \\
\hline$>4,5$ & $5(29,4)$ & $10(52,6)$ & & \\
\hline Total & $17(100)$ & $19(100)$ & & \\
\hline \multicolumn{5}{|l|}{ Estudio al Fresco } \\
\hline Células Clue/Gv & $2(11,8)$ & $2(10,5)$ & 0,906 & $0,986(0,782-1,244)$ \\
\hline Otros hallazgos & $15(88,2)$ & $17(89,5)$ & & \\
\hline Total & $17(100)$ & 19(100) & & \\
\hline \multicolumn{5}{|l|}{ Cítologia Vaǵinal } \\
\hline Gv & $1(5,9)$ & $2(10,5)$ & 0,615 & $0,559(0,055-5,627)$ \\
\hline Otros hallazgos & $16(94,1)$ & $17(89,5)$ & & \\
\hline Total & $17(100)$ & $19(100)$ & & \\
\hline
\end{tabular}

Grupo B: 2 g por 1 día; Grupo C: 2 g por dos días. 
El tinidazol es una prodroga que es convertida a su forma activa in vivo; tiene un bajo peso molecular y penetra la membrana celular de las bacterias aeróbicas y anaeróbicas. Después de penetrar en la bacteria susceptible, el grupo nitro del tinidazol es convertido en radicales más pequeños pero tóxicos, por un sistema de transporte mediado por una ferridoxina. Se piensa que estos radicales tóxicos se unen al ADN ocasionando daños a este, lo cual conlleva a la muerte de la bacteria.

Al comparar farmacológicamente $\mathbf{5 0 0}$ mg de tinidazol con $500 \mathrm{mg}$ de metronidazol, usados oralmente, el tinidazol tiene una Concentración Máxima (Cmx) más elevada, un área bajo la curva y una vida media más larǵa que el metronidazol. Ambas drogas alcanzan un buen nivel de concentración, 70 a $100 \%$, en los tejidos del tracto genital femenino. Sin embargó, el nivel de la concentración sérica del tinidazol, seguida a la administración oral, es más alto que el del metronidazol. Las propiedades farmacocinéticas de ambas drogas son similares después de la administración oral de $2 \mathrm{~g}$ por VO y de $800 \mathrm{mg}$ y $1600 \mathrm{mg}$ por vía endovenosa; se unen solo en un $12 \%$ a las proteínas plasmáticas y son ampliamente distribuidas en todos los tejidos del organismo. Su eliminación se lleva a cabo por el metabolismo hepático (aproximadamente en un 63\%) $(8,22)$.

La dosis recomendada de los derivados de los 5-nitroimidazolicos tales como el secnidazol y el tinidazol en el tratamiento de la VB es de $2 \mathrm{~g}$ en una sola dosis por 1 a 2 días $(13,15,16)$ excepto el metronidazol; sin embargo, Mohanty y col. (23) publicaron en 1983 una experiencia con el uso de $2 \mathrm{~g}$ de este medicamento en una sola toma con $79 \%$ de curación de la VB. En la mayoría de los estudios relacionados con tinidazol en el tratamiento de la VB se ha usado una sola dosis de 1 a 2 g por 1 a 5 días $(13,23,24)$.

Diferentes estudios comparativos sugieren que el uso del tinidazol por $\mathrm{VO}$ es igualmente eficaz como el uso oral de metronidazol (23,25-29), el uso vaginal de clindamicina $(28,29)$ y del metronidazol en óvulos (30) en el tratamiento de la VB. El uso de $2 \mathrm{~g}$ de tinidazol en dosis única parece ser más efectivo que la dosis única de $2 \mathrm{~g}$ de metronidazol (31), pero ligeramente menos efectivo que el uso del metronidazol por 7 días (24). Schwebke y col. (24) publicaron que no encontraron que el tinidazol fuera más eficaz que el metronidazol cuando se compararon $1 \mathrm{~g}$ de metronidazol VO OD por 7 días con tinidazol $1 \mathrm{~g}$ y $2 \mathrm{~g}$ VO OD por 7 días.

La comparación entre los diferentes estudios del uso del tinidazol en VB es dificultoso (8,32-37); sin embargo, los estudios con placebo y controlados ha demostrado un incremento en el porcentaje de cura del tinidazol; los porcentajes de cura varían entre 46 y $90 \%$ cuando se usa $2 \mathrm{~g}$ VO en un solo día, 74\% cuando se usa la misma dosis por 2 días y 91\% cuando se usa $1 \mathrm{~g}$ VO OD por 5 días $(31,34-36)$. Decir que el tinidazol es más eficaz que el metronidazol y otros derivados 5-nitroimidazolicos, es difícil de concluir actualmente (8). Cuando se ha administrado el tinidazol en dosis de $2 \mathrm{~g}$ en una sola toma, se ha demostrado que este tiene un porcentaje de cura más alto que el metronidazol en la misma dosis $(23,28)$. Sin embargo, hoy día se reconoce que la dosis simple de tinidazol no es tan efectiva como la administración prolongada de metronidazol $(13,37)$.

Ekgren y col. (36) en 1988 compararon $2 \mathrm{~g}$ de tinidazol VO en dosis única con $2 \mathrm{~g}$ de tinidazol VO OD por 2 días, encontrando una mayor eficacia usando el último esquema. Nailor y col. (38) en el 2007 mencionaron que $2 \mathrm{~g}$ de tinidazol $\mathrm{VO}$ en una sola toma por 2 días parece ser más efectiva que la dosis única por VO de 2 g por 1 día, igualmente reportaron que el uso de dosis de $1 \mathrm{~g}$ VO OD por 5 días resulta mucho más efectiva. Armstrong y col. (8) describieron que el uso por varios días es mucho mejor que el uso de dosis única; sin embargo, Livengood y col. (32) concluyeron que el uso de $1 \mathrm{~g}$ VO OD de tinidazol por 5 días y de $2 \mathrm{~g}$ VO OD por 2 días tienen un porcentaje de cura sig-

Vol. 61(1): 5 - 18, 2020 
nificativamente más alto que el placebo en el tratamiento de la VB.

El lineamiento nacional inǵlés del año 2006 para tratamiento de la VB (39) recomienda el uso del metronidazol como tratamiento de primera línea, principalmente por el costo más económico, además recomiendan el uso de $2 \mathrm{~g}$ de tinidazol en una sola dosis como tratamiento secundario o alternativo. Los lineamientos del 2004 para el manejo de enfermedades de transmisión sexual para el tratamiento de la VB, del Colegio Austral-Asiático de Médicos de la Salud Sexual establecen el uso de $2 \mathrm{~g}$ VO en única dosis (40).

El presente reporte preliminar demostró que el uso de $2 \mathrm{~g}$ VO de tinidazol por 1día es igualmente eficaz y curativo de la VB que el uso de 2 g por 2 días. La respuesta de las pacientes del grupo B demostró que una dosis única de $2 \mathrm{~g}$ del tinidazol es curativa de la $\mathrm{VB}$, opuesto a lo mencionado por otros autores $(13,37)$ ya que se encontró que hay una respuesta favorable en las variables $\mathrm{KOH}$, $\mathrm{pH}$, estudio al fresco y CGV. Así mismo, el estudio demuestra que el uso único de $1 \mathrm{~g}$ no es suficiente para lograr la curación clínica y/o citológica de la VB.

Uno de los problemas que confrontó el estudio fue que algunas pacientes no acudieron exactamente a la semana de ingerir el tratamiento; sin embargo, fueron incluidas independientemente de la respuesta al tratamiento y en el grupo al cual se incluyeron. De igual manera, solo hubo un observador con el objeto de evitar los errores interobservador.

En relación a los efectos secundarios, este reporte preliminar no encontró ninguna ventaja en el uso de dosis más bajas y por menos tiempo. Diferentes autores han reportado que no hay ninguna diferencia entre los diferentes derivados 5-nitroimidazolicos en la generación de los diferentes efectos secundarios típicos de estos medicamentos, con respecto a la dosis y la duración $(15,16,24)$.
En conclusión, este reporte preliminar demostró que el uso de $2 \mathrm{~g}$ en dosis única fue tan efectivo como la dosis de $2 \mathrm{~g}$ por 2 días, pero no así, la dosis menor, de $1 \mathrm{~g}$ en dosis única. A pesar que los efectos secundarios no tuvieron que ver con la dosis, el uso de menor tiempo de los derivados 5-nitroimidazolicos favorecería el cumplimiento del tratamiento. En la actualidad estamos trabajando con más casos para aumentar el tamaño de la muestra y llegar a conclusiones definitivas.

\section{REFERENCIAS}

1. Rekha S, Jyothi S. Comparison of visual, clinical and microbiological diagnosisof symptomatic vaginal discharge in the reproductive age group. Int J Pharm Biomed Res 2010; 1(4): 144-148.

2. Kenyon C, Colebunders R, Crucitti T. The global epidemiology of bacterial vaginosis: a systematic review. Am J Obstet Gynecol 2013; 209(6): 505-523.

3. Ma L, Su J, Su Y, Sun W, Zeng Z. Probiotics administered intravaginally as a complementary therapy combined with antibiotics for the treatment of bacterial vaginosis: a systematic review protocol. BMJ 2017; 7(10): e019301. doi: 10.1136/ bmjopen-2017-019301.

4. Paladine HL, Desai UA. Vaǵinitis: diaǵnosis and treatment. Am Fam Physician 2018; 97(5): 321-329.

5. Verstraden $H$, Verhelst $R$, Vaneechoutte $M$, Temmerman M. The epidemiology of bacterial vaginosis in relation to sexual behavior. BMC Infect Dis 2010; 81:10.

6. Walker J, Hocking JS, Fairly CK, Tabrizi S, Chen M, Bowden F, Gunn J, Donovan B, Kaldor J, Bradshaw C. The prevalence and incidence of bacterial vaginosis in a cohort of young Australian women. In: Conference Proceedings of the International Society for Sexually Transmitted Research. Quebec, Canada. 2011; 87(S1): A110-A111.

7. Eschenbach DA, Hillier S, Critchlow C, Stevens C, DeRouen T, Holmes KK. Diaǵnosis and clinical manifestations of bacterial vaginosis. Am J Obstet Gynecol 1988; 158(4): 819-828. 
8. Armstrong N, Wilson JD. Tinidazole in the treatment of bacterial vaginosis. Int $\mathrm{J}$ Women Health 2009; 1: 59-65.

9. Schwebke J. Bacterial Vaǵinosis. Curr Infect Dis Rep 2000; 2(1): 14-17.

10. Lewis PM, Bernstein KT, Aral SD. Vaginal microbiome and its relationship to behavior, sexual health, and sexual transmitted diseases. Obstet Gynecol 2010; 129(4): 643-654.

11. Reiter S, Kellog SS. Bacterial vaǵinosis: a primer for clinicians. Postǵrad Med 2019; 131(1): 8-18.

12. Center for Disease Control and Prevention. 2015 STD Treatment Guidelines - Bacterial Vaǵinosis - Updated diaǵnostic, treatment, and screening recommendations for STDs. Disponible en: https://www.cde.gov/std/ bv/default.htm. Revisado:14/06/2018.

13. Amsel R, Totten PA, Spiegel CA, Chen KCS, Eschenbach DA, Holmes KK. Nonspecific vaǵinitis: diaǵnostic and microbial and epidemiological associations. Am J Med 1983; 74(1): 14-22.

14. Livengood CH. Bacterial vaǵinosis: an overview for 2009. Rev Obstet Gynecol 2009; 2(1): 28-37.

15. Hillier SL, Nyirjesy $P$, Waldbaum AS, Schwebke JR, Morgan FG, Adetoro NA, Braum CJ. Secnidazole treatment of bacterial vaginosis: A trial. Obstet Gynecol 2017; 130(2): 379-386.

16. Núñez JT, Gómez G. Low-dose secnidazole in the treatment of bacterial vaginosis. Int J Gynaecol Obstet 2005; 88(3): 281-285.

17. Raja IM, Basavareddy A, Mukherjee D, Meher BR. Randomized, double-blind, comparative study of oral metronidazole and tinidazole in treatment of bacterial vaginosis. Ind J Pharmacol 2016; 48(6): 654-658.

18. Bohbot JM, Vicaut E, Fagnen D, Brauman M. Treatment of bacterial vaginosis: a multicenter double-blind, double-dummy, randomized phase III study comparing secnidazole and metronidazole. Inf Dis Obstet Gynecol 2010.Epub 2010 Sep 15. http:// dx.doi.org/10.1155/2010/705692.

19. Verstraelen H, Verhelst R, Roelens K, Temmeramn M. Antiseptics and disinfectants for the treatment of bacterial vaginosis: A systematic review. BMC Infect Dis 2012; 12 : 148.
20. Petrina MAB, Consentino LA, Rabe LK, Hillier SL. Susceptibility of bacterial vaginosis (BV)-associated bacteria to secnidazole compared to metronidazole, tinidazole and clindamycin. Anaerobe 2017; 47: 115-119.

21. Thellin O, Zorzi W, Zorzi D, Delvenne P, Heinen E, Elmoualij B, Quatresooz P. Lysozyme as cotreatment during antibiotic use aǵainst vaǵinal infections: a in vitro study on Gardnerella vaǵinalis biofilm models. Int Microbiol 2016; 19(2): 101-107.

22. Nord CE, Kager L. Tinidazole-microbiology, pharmacology and efficacy in anaerobic infections. Infection 1983; 11(1); 54-60.

23. Mohanty KC, Deighton R. Comparison of 2 g single dose of metronidazole, nimorazole and tinidazole in the treatment of vaginitis associated with Gardnerella vaginalis. J Antimicrob Chemother 1987; 19(3): 393-399.

24. Schwebke JR, Desmond RN. Tinidazole versus metronidazole for the treatment of bacterial vaginosis. Am J Obstet Gynecol 2011; 204(3): 211.e1-211.e6.

25. Sanz-Sanz F, Hernandez AD, Sanchez EA, Alvarez CG, Muelas M, Rodriǵuez-Noriega A. Controlled comparative study of metronidazole (Flaǵyl) versus tinidazole (Tricolan) in the treatment of nonspecific vaginitis (vaginitis due to Gardnerella vaginalis). Rev Gynaecol Obstet 1985; 44 (2): 717-720.

26. Buranawarodomkul $P$, Chandeying $V$, Sutthijumroon S. Seven day metronidazole versus sinǵle dose tinidazole as therapy for nonspecific vaginitis. J Med Assoc Thai 1990; 73 (3): 283-287.

27. Sanz-Sanz F, Arreaza L, Vidriales I, Miranda P, Grande J, Valle R. Concerning the different therapeutic options for bacterial vaǵinosis. Proǵress Obstet Ginecol 1996; 39(5): 669-674.

28. Milani M, Barcellona E, Agnello A. Efficacy of the combination of $2 \mathrm{~g}$ oral tinidazole and acidic buffering vaǵinal gel in comparison with vaginal clindamycin alone in bacterial vaǵinosis: a randomized, investigator-blinded controlled trial. Eur J Obstet Gynaecol Reprod Biol 2003; 109(1): 67-71.

29. Milani M, Baldoni A, Guerro B, Spinillo A. The "talent" multicenter trial: efficacy of tinidazole, metronidazole, and tinidazole + acidic gel in the treatment of $\mathrm{B}$. vaginosis. Abstracts of the 86th World Congress for

Vol. 61(1): 5 - 18, 2020 
Infectious and Immunological Diseases in Obstetrics and Gynaecology, 2003. Venice, Italy; 39: 669-674.

30. Schindler EM, Thamm H, Ansmann EB, Sarnow E, Schindler AE. Treatment of bacterial vaǵinitis. Multicenter, randomized, open study with tinidazole in comparison with metronidazole. Fortschr Med 1991; 109(5): 138-140.

31. Ambika B, Mamatha KR, Shivamurthy G. Single dose metronidazole, tinidazole and ornidazole in the treatment of bacterial vaǵinosis - a comparative study. Int $\mathrm{J}$ Basic Clin Pharmacol 2016; 5(5): 1966-1971.

32. Livengood CH, Ferris DG, Wiesenfeld HC, Hillier SL, Soper DE, Nyirjesy P, Marrazzo J, Chatwani A, Fine P, Sobel J, Taylor SN, Wood L, Kanalas JJ. Effectiveness of two tinidazole regimens in treatment of bacterial vaginosis: a randomized controlled trial. Obstet Gynaecol 2007;110 (2): 302-309.

33. Paavonen J, Vesterinen E, Purola E, Jokipii AM, Jokipii L, Holttinen K, Vartiainen E. Single dose of tinidazole in the treatment of vaginal dischargé. Scand J Urol Nephrol Suppl 1984; 86: 237-240.

34. Piot P, Van Dyck E, Godts P, Vanderheyden J. A placebo-controlled, double-blind comparison of tinidazole and triple sulfonamide cream for the treatment of nonspecific vaginitis. Am J Obstet Gynecol 1983; 147(1): 85-89.
35. Van Der Meijden WI. Treatment of nonspecific vaginitis with a single dose of tinidazole. Scand J Infect Dis Suppl 1983; 40: 85-89.

36. Ekgiren $\mathbf{J}$, Norling $\mathbf{B}$, Degre $\mathbf{M}$, Midret $\mathbf{T}$. Comparison of tinidazole given as a single dose and on 2 consecutive days for the treatment of nonspecific bacterial vaginosis. Gynecol Obstet Invest 1988; 26(4): 313-317.

37. Joesoef MR, Schmid GP. Bacterial vagínosis: review of treatment options and potential clinical indications for therapy. Clin Infect Diseases 1995, 20(Supl 1): s72-s79.

38. Nailor MD, Sobel JD. Tinidazole for bacterial vaginosis. Expert Opin Investig Drugs 2007; 16(5):743-751.

39. British Association for Sexual Health and HIV. United Kingdom National Guideline for the management of bacterial vaginosis 2012. Disponible from: https:// www.bashh-guidelines.org/current-guidelines/vaǵinal-discharǵe/bacterial-vaǵinosis2012/. Revisado: 15/05/2019.

40. Australasian College of Sexual Health Physicians. Clinical Guidelines for the management of sexually transmissible infections among priority populations 2018. Disponible from: http://www.sti.guidelines.org.au/sexuallytransmissible-infections/infections-associated -with-sex/bacterial-vaginosis\#management. Revisado:15/05/2019. 